\title{
Physiotherapy Interventions Including Frequent Changes of the Body Position and Stimulation to Physical Activity for Infants Hospitalised with Bronchiolitis: A Feasibility Study of a Randomised Control Trial
}

Sonja Andersson Marforio ( $\square$ sonja.andersson_marforio@med.lu.se )

Lund University: Lunds Universitet https://orcid.org/0000-0003-0122-763X

Annika Lundkvist Josenby

Lund University Faculty of Medicine: Lunds universitet Medicinska fakulteten

Christine Hansen

Skånes universitetssjukhus Lund: Skanes universitetssjukhus Lund

Eva Ekvall Hansson

Lund University Faculty of Medicine: Lunds universitet Medicinska fakulteten

Research

Keywords: feasibility studies, physical therapy modalities, bronchiolitis, randomized controlled trials

Posted Date: May 6th, 2021

DOI: https://doi.org/10.21203/rs.3.rs-490376/v1

License: (9) This work is licensed under a Creative Commons Attribution 4.0 International License. Read Full License 


\section{Abstract}

Background: The effect of a treatment that includes frequent changes of the body position for infants with bronchiolitis has not been evaluated, although it is often used in Swedish hospitals. Because of this, a randomised control trial (RCT) has begun with the aim to evaluate this treatment, comparing the effect of an individualised physiotherapy intervention, a non-individualised intervention, and a control group receiving standard care. The objective of this internal pilot study was to address uncertainties concerning the ongoing RCT and to determine whether the trial is feasible or not, possibly with adjustments to the protocol.

Methods: Descriptive analyses of the recruitment, retention, data supply for the primary end point and the usability of the primary outcome measure were performed. A safety analysis was conducted by an independent analysis group.

Results: Ninety-one infants were included, 33 (36.3\%), 28 (30.8\%), and $30(33.0 \%)$ in the respective allocation groups. Fifty-nine (64.8\%) were boys. The median age was 2.5 (min-max: 0.2-23.7) months. They remained in the study for a median of 46 hours (min-max: 2-159). The recruitment rate was $19 \%$. The data supply for the primary end point and for the primary outcome measure was lower than anticipated in the original sample size calculation. Difficulties concerning utilising the primary outcome measure were identified. The safety analysis detected no risks of harm related to participation in the study.

Conclusions: It is feasible to continue the RCT with modifications of the analysis plan. Participation in the study was not associated with any safety risks.

Trial registration: ClinicalTrials.gov NCT03575091. Registered 2 July 2018. Retrospectively registered, https://clinicaltrials.gov/ct2/show/NCT03575091?term=NCT03575091\&rank=1

\section{Key Messages Regarding Feasibility}

- The main uncertainties concerned recruitment, retention, data collection, the primary outcome measure, and the safety for participants.

- The recruitment and retention rates were low, which affected the data supply at the chosen time of analysis. The primary outcome measure was not feasible for use in the full RCT. No harmful outcome was detected.

- It is feasible to continue the RCT with some adjustments to the analysis protocol. It is safe to participate in the study.

\section{Background}

Lower respiratory tract infections such as bronchiolitis or pneumonia is the most common reason for infants around the world to become hospitalised (1). The reason for hospitalisation is often respiratory 
distress following increased mucus production and oedema in the smaller airways, and subsequent feeding difficulties $(2,3)$. The treatment in hospitals is mostly supportive, and most patients are treated with supplemented oxygen and fluid (4), and the use of high flow nasal cannula (HFNC) is extensive (5). Some infants may need treatment at an intensive care unit (ICU) (6). To support evacuation of bronchial secretion, reduce respiratory distress and increase oxygenation, physiotherapy (PT) treatment is sometimes used (7-10).

The evidence about the effect of PT treatment for infants hospitalised with acute bronchiolitis or pneumonia is unclear (11) or PT is not generally recommended (12). However, there is a great variety in the described PT treatment methods and their evaluation methods, so guidelines are not applicable for all PT treatment methods. Our research group conducted a survey study to understand and describe current Swedish practices (13), which comprise different measures, but usually with a focus on frequent changes of the body position and stimulation to physical activity. To our knowledge, this treatment for the patient group has not previously been described or evaluated.

To evaluate this treatment, the research group started a randomised control trial (RCT). The main purpose of the RCT is to evaluate the effect of PT treatment in two intervention groups compared to a control group receiving standard care. The details of the RCT are described in a study protocol (14). There were, however, uncertainties about the feasibility of completing the RCT which we decided to evaluate in this study, as is also recommended in the Medical Research Council's (MRC) guidelines on complex interventions (15) and that are further explained by Craig et al. (16) and Richards and Hallberg (17). They stress that it is important to acknowledge and to structure the research process in different steps in order to produce valid results. In the feasibility and piloting step, according to the MRC's guidelines and others $(15,18)$, the researchers test the procedures, the outcome measure, estimate recruitment and retention, and examine the eligibility criteria. Hence, in order to produce high quality research, and in the light of a well-designed study being less likely to produce research waste (19), testing the feasibility of the full RCT was warranted. The aim of this study was to address uncertainties concerning the ongoing RCT and to determine whether the trial is feasible or not, or what adjustments to the protocol are needed.

\section{Methods}

We aimed at making a critical analysis of the feasibility of the protocol concerning recruitment, retention, the chosen point for the primary analysis, and the primary outcome measure, as well as perform a safety analysis, following the intention of the study protocol (14).

\section{Trial design}

This is an internal pilot study (20) conducted in an ongoing clinical two-centre individually randomised controlled trial with three parallel groups. We designed the study with inspiration from literature on the subject $(15,18,21,22)$. The results are reported using the CONSORT extended guideline for pilot and feasibility trials (23), as recommended by Thabane et al. (24). 


\section{Inclusion of participants}

The participants were identified and recruited by the staff in the paediatric wards of two hospitals in the south of Sweden. Inclusion criteria were as follows: age 0-24 months, hospitalised on the basis of acute airway infection, born in gestational week 35 or later. Patients had to be included within 24 hours of hospital admission. At least one of the parents/guardians had to understand written Swedish, English, Arabic or Persian. Exclusion criteria: previous respiratory or cardiac diagnoses. The participants were included between November 2017 and March 2020. The seasonal inclusion period was typically between November and April. In order to admit their child into the study, the parent/s signed a written consent form. The participation in the study ended when the infants were either discharged to home, referred to an ICU or when the parents decided to withdraw the participation of their child.

\section{Interventions}

Details about the interventions are provided in the study protocol (14). The participants were randomised to an individualised physiotherapy intervention, a non-individualised intervention, or a control group. A statistician independent of the research group performed the randomisation, stratified by the two sites, and prepared opaque paper envelopes. The staff in care of the recruited participant at the ward opened the top envelope in the study binder to reveal the allocation group.

All three groups received the standard care at the ward, and the two intervention groups received additional treatment, including different movements of the body. The standard care comprised information to the parents about the importance of fluid intake for their infant, oxygen supplementation, nose drops and suctioning, high flow nasal cannula (HFNC), inhalations, fluid supplementation, and analgesics, according to need. The individualised intervention was performed by a physiotherapist at least once daily. The PT was sitting on a large ball, firmly supporting the infant in different body positions, while bouncing, in order to affect the respiratory pattern of the infant. The FT also stimulated to active movements according to the infant's ability and could choose additional treatments. The nonindividualised intervention was performed by the nursing staff at least once shortly after inclusion and comprised changes of the body position mainly out the bed, but not using the ball, and slightly less variation of activities. After the first 20-minute intervention, the parents in both intervention groups were instructed to continue the movements regularly throughout the day.

\section{Outcomes}

Assessments were made at baseline, after 20 minutes (directly following the first intervention), and every subsequent third hour. The primary outcome measure in the RCT is a composite index that the research group constructed, based on factors that determine if an infant needs hospitalisation (25). The composite index comprises levels of oxygen saturation, supplemented oxygen concentration, high nasal flow treatment, and oral fluid intake (as opposed to tube feeding).

The secondary outcome measures include the Wang score (26), standard vital signs, the parents' observations (on general condition and food intake), time spent at the hospital ward, and referrals to an 
intensive care unit.

According to the analysis plan for the full RCT, baseline assessments will be compared with the assessments after 24 hours. We have also planned to examine any immediate effect of the first intervention, after 20 minutes.

The recruitment was analysed in four different ways: the proportion of included participants, the estimated time to reach target sample size, time to inclusion, and possible improvement before inclusion.

The proportion of included participants was assessed by comparing the number of participants included in the study with the number of patients admitted to the wards during the three winter seasons 20172018, 2018-2019 and 2019-2020 recorded with the International Statistical Classification of Diseases and Related Health Problems - Tenth Revision (ICD-10) diagnoses of respiratory infections J10.0, J11.0, J12.0, J13.0, J14.0, J15.0, J18.0, and J21.0 with subgroups. We additionally assessed what proportion of the infants admitted to one of the hospital wards with the diagnoses above met the inclusion criteria, by reading the medical records of admitted patients at four weeks during the peaks of the Respiratory Syncytial Virus (RSV) infection in Sweden according to national reports (27). The selected weeks were February 26 to March 4 and March 19 to 25 in 2018 and February 11 to 24 in 2019. We further estimated how long it would take to include 162 patients (primary sample size calculation) in the study, based on how many had been included during these three winter seasons.

Time to inclusion was calculated by comparing the time for admission to the ward (recorded in the local patient administrative system) to the time for the baseline assessment. This item was reported in hours.

A possible improvement before inclusion was analysed to examine the inclusion criteria of admitted delay for 24 hours after admittance to the ward. The possibility to detect any changes from baseline and between groups may decline with increasing time if the participants might already have recovered substantially before inclusion. This was analysed as follows: the infants included after the median time to inclusion were further analysed. Those who received oxygen supplementation or HFNC at the first assessment were excluded as they were considered still severely affected, and the delay was thus judged as acceptable. For the infants who did not have oxygen supplementation or HFNC at inclusion, we compared levels of oxygen saturation, respiratory rate, and heart rate at admittance to the ward with values at inclusion. If the values had changed from one level to another in the Rapid Emergency Triage and Treatment System - pediatric (RETTS-p) (28-31), which is commonly used in Swedish hospitals, the change was considered as clinically significant and more problematic for the study. Additionally, and in the same way, we analysed change in RETTS-p level among the infants who were included by mistake after 24 hours at the ward, and who thus did not fulfill the inclusion criteria.

Retention was primarily analysed by calculating how many hours the participants remained in the study. We further analysed the proportion of participants still in the study with recorded data about saturation or heart rate at hour 24 (primary end point), hour 36, hour 48, and longer. We analysed data from these occasions to examine participant retention at the chosen point for analysis, and the feasibility of delaying 
the primary end point. Our theory was that the possibility to detect changes from baseline and analyse any differences between the groups would improve the closer to discharge the analysis was made. Based on the previous sample size calculation and the planned analysis for the full RCT, a retention rate of $85 \%$ or more was considered acceptable.

Primary outcome measure. The proportion of complete reported data in the composite index at baseline and at hour 24 was analysed using frequency measures. Each of the four domains in the index was analyzed separately, i.e. level of oxygen saturation, concentration of supplemented oxygen, level of supplied high nasal air flow, and oral fluid intake as a proportion of calculated daily need. A level of $85 \%$ was determined as sufficient, following the previous sample size calculation. We additionally critically reviewed the quality of the collected data in the composite index in order to determine usability.

The safety analysis was performed by an independent group consisting of a statistician and a paediatrician. Following the instructions from the research group, they thoroughly reviewed the data, searching for values that in a clinical setting would necessitate extra treatment or dramatically change the treatment for an infant. The values included in the safety analysis were oxygen saturation, respiratory rate, oxygen supplementation, high nasal air flow, oral fluid intake, length of hospital stay, referrals to ICU, and deaths. They analysed values from the total study population. If values indicating any safety risks had been discovered, they would have continued by analysing the data divided into the separate intervention groups. If they had identified any safety risks, the research group would, after clinical reasoning, either have changed the protocol or terminated the study.

\section{Sample size and statistical methods}

The sample size calculation for the full RCT showed that 162 participants need to be included, 54 in each group. Safety and interim analyses were planned to be performed after inclusion of $50 \%$ of the required participants, and this feasibility study was performed at this stage accordingly (14). The safety analysis group was blinded regarding allocation group for the participants. Blinding of parents to participants, care providers, or assessors was not possible due to the nature of the intervention. To minimise possible bias, one person performed the intervention, and another person made the assessment.

We used the IBM SPSS Statistics 27 Windows (IBM Corporation, Armonk, NY, USA) for analyses of the data. The analyses were descriptive, using median with min-max (IQR), and numbers with percentages when appropriate.

\section{Results}

For a description of the participant flow, see Fig. 1. Of the 91 included participants, 11 did not start any intervention or assessment for different reasons and were thus drop-outs after randomization. 
Table 1

Baseline characteristics of the included participants, $n=91$

\begin{tabular}{|c|c|c|c|c|}
\hline & $\begin{array}{l}\text { Control } \\
\text { group } n= \\
30\end{array}$ & $\begin{array}{l}\text { Non-individualized } \\
\text { intervention } n=28\end{array}$ & $\begin{array}{l}\text { Individualized } \\
\text { intervention } n=33\end{array}$ & $\begin{array}{l}\text { Total sample } n \\
=91\end{array}$ \\
\hline $\begin{array}{l}\text { Gender n (\%) } \\
\text { male/female }\end{array}$ & $\begin{array}{l}21(67.7) \\
/ 9(30.0)\end{array}$ & $15(54.6) / 13(46.4)$ & $\begin{array}{l}23(69.7) / 10 \\
(30.3)\end{array}$ & $\begin{array}{l}59(64.8) / 32 \\
(35.2)\end{array}$ \\
\hline $\begin{array}{l}\text { Age median (min- } \\
\text { max) months }\end{array}$ & $\begin{array}{l}3.3(0.2- \\
22.4)\end{array}$ & $2.9(0.5-22.2)$ & $2.1(0.3-23.7)$ & $\begin{array}{l}2.5(0.2-23.7) \\
\text { IQR* }^{*} 1.2,6.9\end{array}$ \\
\hline \multicolumn{5}{|l|}{$\begin{array}{l}\text { Infectious agent } \mathrm{n} \\
(\%)^{\star \star}\end{array}$} \\
\hline 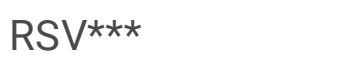 & $18(60.0)$ & $19(67.9)$ & $22(66.7)$ & $59(64.8)$ \\
\hline influenza & $2(6.7)$ & $3(10.7)$ & $4(12.1)$ & $9(9.9)$ \\
\hline other & $1(3.3)$ & $3(10.7)$ & $3(9.1)$ & $7(7.7)$ \\
\hline negative & $5(16.6)$ & $1(3.6)$ & $1(3.0)$ & $7(7.7)$ \\
\hline missing & $5(16.6)$ & $2(7.1)$ & $4(12.1)$ & $11(12.1)$ \\
\hline \multicolumn{5}{|l|}{$\begin{array}{l}\text { Heredity atopic } \\
\text { disease } \mathrm{n}(\%)\end{array}$} \\
\hline asthma & $10(33.3)$ & $10(35.7)$ & $12(36.4)$ & $32(35.2)$ \\
\hline $\begin{array}{l}\text { other (allergies, } \\
\text { eczema etc) }\end{array}$ & $7(23.3)$ & $5(17.9)$ & $11(33.3)$ & $23(25.3)$ \\
\hline none & $10(33.3)$ & $10(35.7)$ & $3(9.1)$ & $23(25.3)$ \\
\hline missing & $3(10.0)$ & $3(10.7)$ & $7(21.2)$ & $13(14.3)$ \\
\hline \multicolumn{5}{|l|}{$\begin{array}{l}\text { Passive smoking } \\
\text { exposure }\end{array}$} \\
\hline no & $22(73.3)$ & $21(75.0)$ & $17(51.6)$ & $60(65.9)$ \\
\hline yes & $5(16.6)$ & $3(10.7)$ & $8(24.2)$ & $16(17.6)$ \\
\hline missing & $3(10.0)$ & $4(14.3)$ & $8(24.2)$ & $15(16.5)$ \\
\hline \multicolumn{5}{|c|}{ *IQR = Interquartile range } \\
\hline$\star \star$ two individuals $\mathrm{h}$ & d positive te & for both RVS and infle & & \\
\hline
\end{tabular}

Outcomes of the feasibility study are reported below. 
During the stated four weeks in 2018 and 2019, 46 infants were hospitalised with the selected diagnoses of respiratory infections. Twenty-nine infants (63\%) met the inclusion criteria and $17(37 \%)$ did not, due either to age $(n=12)$, heart disease $(n=2)$, asthma $(n=2)$ or premature birth $(n=1)$. During the entire study period, 762 infants were admitted to the two wards with the selected diagnoses of respiratory infections. By using the results from the in-depth examination above on the whole sample $(63 \%$ estimated to meet the inclusion criteria), 480 patients remained eligible. During the study period, 91 infants were included, which constitutes an inclusion rate of $19 \%$. Parents of 33 infants (7\%) were recorded to have declined participation. The number of parents not asked to participate is not known in detail, but the number of those recorded to have been missed is displayed in the flow chart in Fig. 1.

In the winter season 2017-2018, 33 participants were included, in the season 2018-2019, 27 participants, and in the season 2019-2020, 31 participants. If the inclusion rate follows the same pace, an additional 2.5 winter seasons will be needed to reach the original calculated sample size.

For the 80 infants who started assessments and interventions, participation began at median 13 hours (min-max: 0-24 hours, IQR: 6, 18) after admission to the hospital wards.

Twenty-three infants without supplemented oxygen or HFNC were included after 13 hours and were analysed regarding vital signs. Six of them had improved regarding RETTS-p before inclusion in the study, and one had deteriorated. Individual data are available in Additional file 1. We additionally analysed 11 individuals who were excluded from the first analysis as they did not meet the inclusion criteria of being included within 24 hours. They were included after a median of 31 hours (min-max 2536 hours). We omitted four infants who received oxygen supplementation or HFNC at inclusion, and seven remained. Six of these had improved and one had remained on the same RETTS-p level. Individual data are available in Additional file 2. Six of 91 infants in the study and six of 11 who were included after 24 hours were shown to improve significantly before inclusion.

Retention and point for analysis

The participants remained in the study for a median of 46 hours (min-max: 2-159 hours, IQR: 22, 71), calculated on the 80 participants who started their intervention/control.

The proportions of all included participants $(n=91)$ still in the study with data on saturation or heart rate at 24,36 and 48 hours are displayed in Table 2 . For 33 infants (36.3\%) some incomplete data was also reported after 48 hours. 
Table 2

Participant retention and data supply at different points for follow-up $\mathrm{n}=91$

\begin{tabular}{|c|c|c|c|}
\hline & $\begin{array}{l}24 \mathrm{~h} \\
\mathrm{n}(\%)\end{array}$ & $\begin{array}{l}36 \mathrm{~h} \\
\mathrm{n}(\%)\end{array}$ & $\begin{array}{l}48 \mathrm{~h} \\
\mathrm{n}(\%)\end{array}$ \\
\hline \multicolumn{4}{|l|}{ Saturation } \\
\hline valid & $57(62.6)$ & $42(46.2)$ & $35(38.5)$ \\
\hline discharged & $16(17.6)$ & $25(27.5)$ & $32(35.2)$ \\
\hline drop-outs* & $13(14.3)$ & $13(14.3)$ & $13(14.3)$ \\
\hline missed $\star \star$ & $5(5.5)$ & $11(12.1)$ & $11(12.1)$ \\
\hline 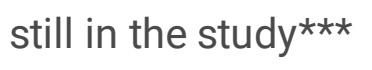 & $62(68.1)$ & $52(57.1)$ & $46(50.5)$ \\
\hline \multicolumn{4}{|l|}{ Heart rate } \\
\hline valid & $57(62.6)$ & $43(47.3)$ & $34(37.4)$ \\
\hline discharged & $16(17.6)$ & $25(27.5)$ & $32(35.2)$ \\
\hline drop-outs* & $13(14.3)$ & $13(14.3)$ & $13(14.3)$ \\
\hline missed $\star \star$ & $5(5.5)$ & $10(11.0)$ & $12(13.2)$ \\
\hline still in the study & $62(68.1)$ & $52(57.1)$ & $46(50.5)$ \\
\hline \multicolumn{4}{|c|}{$\begin{array}{l}\text { *Drop-outs indicates infants who either did not start the interventions at all }(n=11) \text {, or infants for } \\
\text { whom the parents withdrew their participation after some time }(n=2)\end{array}$} \\
\hline \multicolumn{4}{|c|}{$\begin{array}{l}* * \text { 'Missed' indicates that the score was not filled out at this specific time, but the patient was still at } \\
\text { the ward and there are values possible to impute from the assessments before and/or after this }\end{array}$} \\
\hline \multicolumn{4}{|c|}{$\begin{array}{l}\star \star \star \star \\
\text { values possill in the study' comprises the combined values for 'valid' and 'missed', i.e. recorded data or }\end{array}$} \\
\hline
\end{tabular}

At 24 hours there was less data recorded than needed for the primary analysis based on earlier sample size calculation, and data continued to be lost over increasing time.

Primary outcome measure.

The proportions of complete reported data in the composite index for the participants who started interventions/controls $(n=80)$ are displayed in Table 3 . 
Table 3

Proportion of complete reported data and missing data in the composite index (primary outcome measure), $\mathrm{n}=80$

\begin{tabular}{|c|c|c|}
\hline & Complete n (\%) & Missing n (\%) \\
\hline \multicolumn{3}{|c|}{ Level of oxygen saturation } \\
\hline at baseline & $80(100.0)$ & $0(0.0)$ \\
\hline at $24 \mathrm{~h}$ & $57(71.3)$ & $23(28.7)^{a}$ \\
\hline \multicolumn{3}{|c|}{ Concentration of supplemented oxygen } \\
\hline at baseline & $61(76.3)$ & $19(23.8)^{b}$ \\
\hline at $24 \mathrm{~h}$ & $43(53.8)$ & $37(46.3)^{\mathrm{c}}$ \\
\hline \multicolumn{3}{|c|}{ Level of supplied high nasal flow treatment } \\
\hline at baseline & $80(100.0)$ & $0(0.0)$ \\
\hline at $24 \mathrm{~h}$ & $54(67.5)$ & $26(32.5)$ \\
\hline \multicolumn{3}{|l|}{ Oral fluid intake } \\
\hline the first 24 hours & $25(31.3)$ & $55(68.8)^{d}$ \\
\hline \multicolumn{3}{|c|}{ a due to discharged, missed registration, or drop-outs } \\
\hline \multicolumn{3}{|c|}{ b due to receiving low flow $\mathrm{O}_{2}$ or missed registration } \\
\hline \multicolumn{3}{|c|}{${ }^{c}$ due to low flow $\mathrm{O}_{2}$ supplementation, discharged, missed registration, or drop-outs } \\
\hline d due to incomple & ration, or dischars & \\
\hline
\end{tabular}

At the wards, the oxygen saturation was recorded either with or without oxygen supplementation, which makes it difficult to estimate the severity of illness using the scoring, and when removing participants without supplemented oxygen there was a low level of complete recorded data on this variable $(38.8 \%)$ on the two occasions. At baseline, 32 individuals (40\%) received oxygen supplementation, and at 24 hours $24(30 \%)$ did.

The concentration of oxygen supplementation in the composite index is based on high flow oxygen supplementation and does not include participants with low flow oxygen supplementation. Of the 80 participants who started interventions or controls, 18 (22.5\%) received low flow oxygen supplementation at the first assessment. At 24 hours, 12 of the 80 (15.0\%) received low flow oxygen supplementation.

The proportion of complete recorded data for the primary outcome is lower than anticipated when the sample size for the RCT was calculated. The registration of oxygen saturation for participants both with 
and without supplemented oxygen, as well as the use of high and low flow oxygen supplementation at the wards, further complicates the use of the composite index.

\section{The safety analysis.}

The safety analysis group reported that they did not find values indicating any safety risks associated with participation in the study when analysing the entire study population. Thus, they did not continue to analyse the data divided into the respective intervention groups.

\section{Discussion}

This study was conducted with the aim of assessing the feasibility of on ongoing RCT that will evaluate the common PT praxis in Sweden involving frequent changes of the body position for infants hospitalised with bronchiolitis or other lower respiratory tract infections. Our results show that the data supply for the primary end point and for the primary outcome measure was lower than anticipated in the original sample size calculation. Difficulties concerning utilising the primary outcome measure were identified. The safety analysis detected no risks of harm related to participation in the study. We agree with Chalmers et al. (32) that for scientific and ethical reasons it is important that clinical research is well designed, as it is performed using public funds and involves many people, in this case infants with respiratory infections, their parents, and busy nursing staff, and this study has contributed to increase the feasibility of an ongoing trial.

\section{Objectives}

Our main concerns were whether the recruitment, retention, primary outcome measure, and follow-up point for analysis were feasible for the RCT, and we also wanted to assess possible safety risks.

The outcome of an intervention in an acute hospital setting is not always easy to establish, as is also discussed elsewhere $(33,34)$. The primary outcome measure in the RCT was chosen because of its clinical implication and objectivity. We were not certain, however, about the usability of the composite index and whether it was possible to collect the data successfully. Further, we did not know how long the participants would stay hospitalised. In order to capture as much improvement data as possible, we wanted to make the primary analysis as close to the infants' discharge as possible, so looked for the most appropriate time for analysis. In order not to miss any major clinical improvement before inclusion, we also wanted to analyse the admitted time delay before inclusion.

Recruitment. We did not find previous data on anticipated recruitment rate suitable for this study, and our assessment of the outcome is based on clinical reasoning. We expected many infants to be pre-terms or to have comorbidities, and thus not fulfil the inclusion criteria. The data in this study partly support this theory, but the recruitment rate is even lower than we expected, based on clinical reasoning about the high prevalence of infants with bronchiolitis in hospitals. Some parents obviously rejected the offer to participate in the study, and the reasons for that remain to be studied. However, the most likely 
contributory factor to the low recruitment rate was that many parents were not asked to participate. Many infants with respiratory diagnoses are hospitalised during the night, and we have received informal information from the staff that they were reluctant to ask for participation in the study at those hours so as not to disturb the families or because of working routines. During peaks of the RSV infection, the staff also expressed that they were sometimes too busy to enrol participants.

Admitted delay to enrolment. Some of the infants included in the study within the stipulated time showed a clinically significant improvement before the interventions started, and of the infants included after 24 hours a larger proportion improved before inclusion. This supports our view that it would have been preferable if all participants had been included immediately after admittance to the ward, which unfortunately may prove difficult in clinical reality, as this study has shown. The results in the full RCT may be affected, though in this case to a rather limited extent.

Retention. This study showed that the hospital stay was short, which, together with the drop-outs and missed registrations, resulted in low data supply. These findings were important for us when considering the continuation of the full RCT and may also be interesting for other researchers planning to undertake similar clinical studies. We did not fully anticipate the proportion of missed registrations, as the assessment protocol was constructed together with nursing staff to make it easy to fill out. In order to enhance recruitment and data collection it would probably be helpful to have extra staff present on the sites at all times with the responsibility to recruit participants and support data collection. The analyses in this study on participant retention and data supply does not support a primary analysis at 24 hours, and it will not be feasible to postpone the time for the primary analysis.

Primary outcome measure. Data for the composite index in total was considerably lacking. The data on HFNC and oxygen saturation, however, was complete for all participants in the study at baseline, and ample data supply was retained at hour 24 , so it was feasible to collect these items rather successfully. There was a considerable lack of data in the feeding score, which was somewhat surprising to the research group. For infants who were breast-fed, we learned that the practice at the wards was often to encourage the mothers themselves to put the infant on the scales before and after feeding and note the different weights. This is understandably difficult to undertake at all hours, not least during the night, and might have contributed to the low level of data. There may also have been a pedagogical gap towards the staff about the importance of recording this item properly, as they sometimes marked the paper protocols with an X followed by the explanatory note "breast feeding". Because of the low data supply and the difficulties in utilising the composite index, the primary outcome measure for the full RCT will be changed.

Safety analysis. We agree with loannidis et al. (35) about the importance of assessing and reporting harms in clinical trials. As no safety risks were identified in this study, we do not anticipate any harm to be connected to completing the full RCT.

\section{Suggested changes to the analysis plan.}


Since this study revealed issues related to feasibility that needed to be improved, the following changes for the full RCT are suggested. Based on data from this study (see Additional file 3), we will change the primary outcome measure to 'time to improvement' and the statistical analysis method to Kaplan Meier (time-to-event). The proposed change has the advantage of capturing improvement through the entire hospital stay and will thus not be restricted to one pre-set time (previous 24 hours), which is supported by data in this study. A new sample size calculation will be performed, based on the data collected in this feasibility study.

\section{Limitations}

It was difficult to determine progression criteria or cut-off points for the different outcomes, which is desirable for feasibility studies (19). We have, however, tried to make informed clinical reasoning around this, to compensate for the lack of external guidance. Adding interviews with staff might have provided further information about the difficulties concerning recruitment and data recording, and possibly also pointed towards solutions for these. However, the first author (SAM) has regularly received informal information, although not scientifically structured, from meetings with the contact staff, the management and with all staff together while running the trial.

\section{Conclusions}

It is feasible to continue the full RCT with modifications of the analysis plan. As the study concerns treatment for a large and vulnerable group of patients, it is valuable for clinical as well as ethical reasons to make use of the collected data, continue the ongoing RCT, and evaluate the effect of the PT interventions. Participation in the study was not associated with any safety risks.

\section{Abbreviations}

RCT randomised control trial

RSV respiratory syncytial virus

HFNC high flow nasal cannula

ICU intensive care unit

FT fysiotherapy/fysiotherapist

ICD-10 International Statistical Classification of Diseases and Related Health Problems - Tenth Revision RETTS-p Rapid Emergency Triage and Treatment System - pediatric

IQR interquartile range

h hours 


\section{Declarations}

\section{Ethics approval and consent to participate}

Before inclusion, the parent/s received written and oral information about the study. To approve the participation of their child they signed a written consent form. The principals of the Declaration of Helsinki were followed, and the study was approved by the Swedish Ethical Review Authority (2017/190).

\section{Consent for publication}

None of the published material contains identifiable participant information.

\section{Availability of data and materials}

All data are archived according to the Swedish Act concerning the Ethical Review of Research Involving Humans to attain confidentiality and are available from the corresponding author on reasonable request.

\section{Competing interests}

The authors declare that they have no competing interests

\section{Registration}

The full RCT is registered at ClinicalTrials.gov NCT03575091

\section{Protocol}

The full trial protocol (14) can be accessed through the following link: https://rdcu.be/b7wyE

\section{Funding}

The Academy of Caring Sciences, Skåne University Hospital: 2017 and 2018 contribution to the salary for SAM. Lions' Research grant: 2018 contribution to the salary for SAM while designing the study and for laboratory tests. Skåne University Hospital grant 2018 and 2019: contribution to the salary for SAM. Skåne County Council's Research and Development Foundation 2019 and 2020: contribution to statistician support, the randomisation process, laboratory tests, for translations of information material to guardians of the participants and documents for publication, as well as the salary for SAM. The funders played no part in study design; the collection, management, analysis and interpretation of data; the writing of the report; or the decision to submit the report for publication.

\section{Authors' contributions}

All authors participated in the design of the study. SAM performed the data collection, analysed the data, and drafted the initial manuscript. SAM and EEH wrote the subsequent version of the manuscript. All 
authors read and approved the final version of the manuscript.

\section{Acknowledgements}

The authors would like to thank MD Dr Victoria Palmcrantz and statistician Dr Susann Ullén for performing the safety analysis, and statistician MSc Helene Jakobsson for valuable help with guidance about the statistical analyses. They would also like to thank the parents admitting their children into the study, and the staff who helped with the data collection and interventions.

\section{References}

1. Munoz-Quiles C, Lopez-Lacort M, Ubeda-Sansano I, Aleman-Sanchez S, Perez-Vilar S, Puig-Barbera J, et al. Population-based Analysis of Bronchiolitis Epidemiology in Valencia, Spain. Pediatr Infect Dis J. 2016;35(3):275-80.

2. Eber E, Midulla F. ERS Handbook of paediatric respiratory medicine. 1 ed. Sheffield: The European Respiratory Society; 2013.

3. Caffrey Osvald E, Clarke JR. NICE clinical guideline: bronchiolitis in children. Archives of disease in childhood Education practice edition. 2016;101(1):46-8.

4. Florin TA, Plint AC, Zorc JJ. Viral bronchiolitis. The Lancet. 2017;389(10065):211-24.

5. Lin J, Zhang Y, Xiong L, Liu S, Gong C, Dai J. High-flow nasal cannula therapy for children with bronchiolitis: a systematic review and meta-analysis. Arch Dis Child. 2019;104(6):564-76.

6. Schlapbach LJ, Straney L, Gelbart B, Alexander J, Franklin D, Beca J, et al. Burden of disease and change in practice in critically ill infants with bronchiolitis. Eur Respir J. 2017;49(6):1601648.

7. Gajdos V, Katsahian S, Beydon N, Abadie V, de Pontual L, Larrar S, et al. Effectiveness of chest physiotherapy in infants hospitalized with acute bronchiolitis: a multicenter, randomized, controlled trial. PLoS Med. 2010;7(9):e1000345.

8. Postiaux G, Zwaenepoel B, Louis J. Chest physical therapy in acute viral bronchiolitis: an updated review. Respir Care. 2013;58(9):1541-5.

9. Gomes EL, Postiaux G, Medeiros DR, Monteiro KK, Sampaio LM, Costa D. Chest physical therapy is effective in reducing the clinical score in bronchiolitis: randomized controlled trial. Rev Bras Fisioter. 2012;16(3):241-7.

10. Rochat I, Leis P, Bouchardy M, Oberli C, Sourial H, Friedli-Burri M, et al. Chest physiotherapy using passive expiratory techniques does not reduce bronchiolitis severity: a randomised controlled trial. Eur J Pediatr. 2012;171(3):457-62.

11. Gomes GR, Donadio MVF. Effects of the use of respiratory physiotherapy in children admitted with acute viral bronchiolitis. Archives de Pédiatrie. 2018;25(6):394-8.

12. Roque i Figuls M, Gine-Garriga M, Granados Rugeles C, Perrotta C, Vilaro J. Chest physiotherapy for acute bronchiolitis in paediatric patients between 0 and 24 months old. Cochrane Database Syst Rev. 2016;2:CD004873. 
13. Andersson-Marforio S, Hansen C, Ekvall Hansson E, Lundkvist Josenby A. A survey of the physiotherapy treatment methods for infants hospitalised with acute airway infections in Sweden. Eur J Phys. 2019. https://doi.org/10.1080/21679169.2019.1663925.

14. Andersson-Marforio S, Lundkvist Josenby A, Ekvall Hansson E, Hansen C. The effect of physiotherapy including frequent changes of body position and stimulation to physical activity for infants hospitalised with acute airway infections. Study protocol for a randomised controlled trial. Trials. 2020;21(1):803.

15. Medical research council. Complex interventions guidance [Internet]. [cited 2020 February 24]. Available from: https://mrc.ukri.org/search-results/?keywords=complex+interventions\&siteid=mrc.

16. Craig P, Dieppe P, Macintyre S, Michie S, Nazareth I, Petticrew M. Developing and evaluating complex interventions: the new Medical Research Council guidance. Int J Nurs Stud. 2013;50(5):587-92.

17. Richards DA, Hallberg IR, editors. Complex interventions in health: an overview of research methods. London: Routledge; 2015.

18. Thabane L, Ma J, Chu R, Cheng J, Ismaila A, Rios LP, et al. A tutorial on pilot studies: the what, why and how. BMC Med Res Methodol. 2010;10:1.

19. Ioannidis JPA, Greenland S, Hlatky MA, Khoury MJ, Macleod MR, Moher D, et al. Increasing value and reducing waste in research design, conduct, and analysis. The Lancet. 2014;383(9912):166-75.

20. Lancaster GA, Dodd S, Williamson PR. Design and analysis of pilot studies: recommendations for good practice. Journal of evaluation in clinical practice. 2004;10(2):307-12.

21. Arain M, Campbell MJ, Cooper CL, Lancaster GA. What is a pilot or feasibility study? A review of current practice and editorial policy. BMC Med Res Methodol. 2010;10:67.

22. Eldridge SM, Lancaster GA, Campbell MJ, Thabane L, Hopewell S, Coleman CL, et al. Defining Feasibility and Pilot Studies in Preparation for Randomised Controlled Trials: Development of a Conceptual Framework. PLoS One. 2016;11(3):e0150205.

23. Eldridge SM, Chan CL, Campbell MJ, Bond CM, Hopewell S, Thabane L, et al. CONSORT 2010 statement: extension to randomised pilot and feasibility trials. Bmj. 2016;355:i5239.

24. Thabane L, Hopewell S, Lancaster GA, Bond CM, Coleman CL, Campbell MJ, et al. Methods and processes for development of a CONSORT extension for reporting pilot randomized controlled trials. Pilot Feasibility Stud. 2016;2:25.

25. The National Institute for Health and Care Excellence (NICE). Bronchiolitis in children: diagnosis and management. NICE guideline NG9 [Internet]. Manchester: The National Institute for Health and Care Excellence; 2015 [cited 201916 August]. Available from:

https://www.nice.org.uk/guidance/ng9/chapter/1-Recommendations\#when-to-admit.

26. Wang EE, Milner RA, Navas L, Maj H. Observer agreement for respiratory signs and oximetry in infants hospitalized with lower respiratory infections. Am Rev Respir Dis. 1992;145(1):106-9.

27. Folkhälsomyndigheten. RSV (Respiratory Syncytial Virus) 2018-2019 [Internet]. Solna, Sweden: Public health agency of Sweden (Folkhälsomyndigheten); 2020 [cited 202023 November]. Available 
from: https://www.folkhalsomyndigheten.se/folkhalsorapportering-statistik/statistik-ao/sjukdomsstatistik/rsv/?t=county.

28. Predicare RETTS. Pediatrics [Internet]. Göteborg: Predicare; [cited 202116 April]. Available from: https://rise.articulate.com/share/ygZEg740-N8wM43MjjPSx22Ec0b1TzXf.

29. Magnusson C, Herlitz J, Karlsson T, Jiménez-Herrera M, Axelsson C. The performance of the EMS triage (RETTS-p) and the agreement between the field assessment and final hospital diagnosis: a prospective observational study among children < 16 years. BMC Pediatrics. 2019;19(1):500.

30. Ødegård SS, Tran T, Næss-Pleym LE, Risnes K, Døllner H. A validity study of the rapid emergency Triage and treatment system for children. Scandinavian Journal of Trauma Resuscitation Emergency Medicine. 2021;29(1):18.

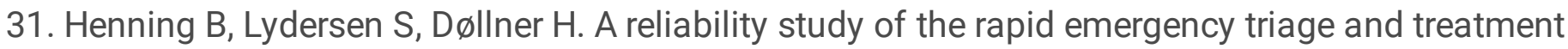
system for children. Scand J Trauma Resusc Emerg Med. 2016;24:19.

32. Chalmers I, Glasziou P. Avoidable waste in the production and reporting of research evidence. Lancet. 2009;374(9683):86-9.

33. Lorenz W, Troidl H, Solomkin JS, Nies C, Sitter H, Koller M, et al. Second step: testing-outcome measurements. World J Surg. 1999;23(8):768-80.

34. Lorenz W, Stinner B, Bauhofer A, Rothmund M, Celik I, Fingerhut A, et al. Granulocyte-colony stimulating factor in the prevention of postoperative infectious complications and sub-optimal recovery from operation in patients with colorectal cancer and increased preoperative risk (ASA 3 and 4). Protocol of a controlled clinical trial developed by consensus of an international study group. Part one: rationale and hypothesis. Inflamm Res. 2001;50(3):115-22.

35. Ioannidis JP, Evans SJ, Gøtzsche PC, O'Neill RT, Altman DG, Schulz K, et al. Better reporting of harms in randomized trials: an extension of the CONSORT statement. Ann Intern Med. 2004;141(10):781-8.

\section{Figures}




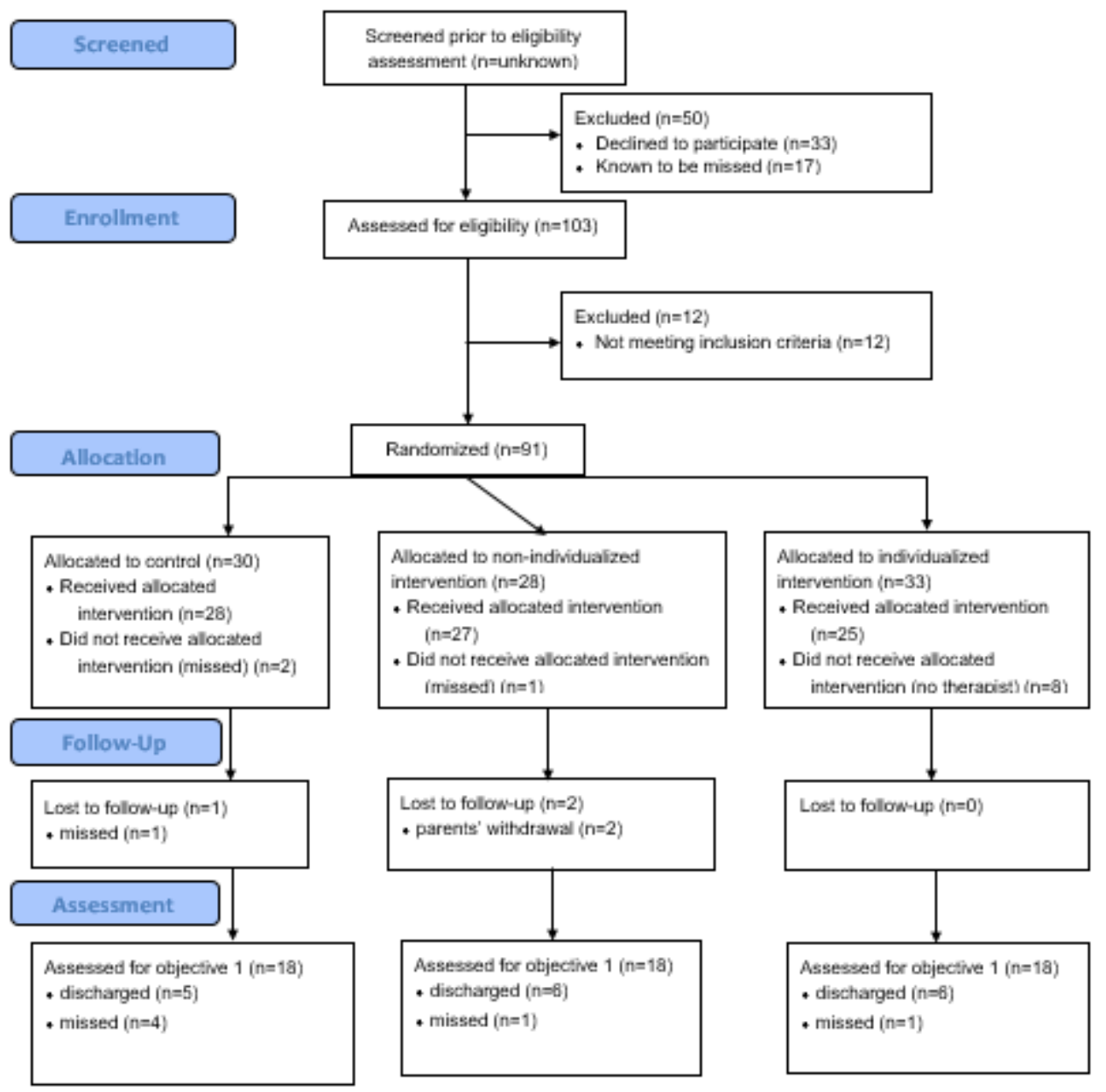

Figure 1

Flow chart of the participant flow

\section{Supplementary Files}

This is a list of supplementary files associated with this preprint. Click to download.

- Additionalfile1.docx

- Additionalfile2.docx

- Additionalfile3.docx

- CONSORTChecklist.doc 